\title{
Ancient frog could spearhead conservation efforts
}

\author{
Newly discovered, this unique creature is already under threat from dam projects.
}

Sir - The discovery of the purple or pignose frog Nasikabatrachus sahyadrensis was recently reported by two groups (see Nature 425, 711-714; 2003, and Current Science 86, 211-216; 2004). The sole member of the family Nasikabatrachidae, this creature evolved some 50 million years earlier than any other known frog in India.

Remarkably, the frog was well known to local people, suggesting that science does not pay enough attention to local knowledge.

This 'new' discovery was the culmination of many field studies on herpetofauna that gained momentum during the 1990s in the
Western Ghats. In a News and Views article, "The coelacanth of frogs" (Nature 425, 669-670; 2003), Blair Hedges decries the policy carried out by some governments of restricting foreigners' access to biodiversity hot spots, and considers this an impediment to the discovery of rare and endangered species.

However, both of the groups who arrived at these results relied minimally on Western expertise and infrastructure. There is still a need for dedicated molecular-genetics facilities for ecology and evolutionary biology in developing countries. Building local capacity and infrastructure may be the best way to accelerate the discovery of new species in remote areas, and this is where responsible scientists must focus their efforts.

The purple frog also has important conservation implications. Inundation of large chunks of valley forests in the Western Ghats by dam projects could spell disaster to this ancient amphibian and many other endemic taxa. Nasikabatrachus can be used as a flagship to promote the conservation of important habitats in the region.

Ramesh K. Aggarwal

Centre For Cellular And Molecular Biology,

Uppal Road, Tarnaka, Hyderabad 500 007, India

\section{Diversity project takes time but reaps rewards}

Sir - Your News Feature on the HapMap project (Nature 425, 758-759; 2003)

characterizes the Human Genome Diversity Project (HGDP) as an "ill-fated exercise" that "never got off the ground". This is not accurate. The birth of the HGDP required time and perseverance because of misunderstandings during its development, which led to a 1997 review by the US National Research Council. This reviewing committee was persuaded of the scientific merit of human genome diversity projects, while also recognizing the ethical and legal challenges implicit in studies of human variation.

Their faith in the HGDP has been rewarded. Since April 2002 a collection of more than 1,000 DNA samples from 51 populations representing most of the world's genome variation has been available to non-profit research laboratories through a collaboration between the HGDP and the Fondation Jean Dausset$\mathrm{CEPH}$ in Paris (see www.cephb.fr/HGDPCEPH-Panel).

All samples used for this resource were collected with proper informed consent; the privacy of the persons volunteering these samples remains protected. Since 1997, the HGDP has developed and promoted the best legal practices available for studying indigenous populations.

Within months of its public release, the HGDP-CEPH resource became the basis of the most comprehensive description of the genetic structure of human populations ever undertaken (N. A. Rosenberg et al. Science 298, 2381-2385; 2002). This publication, which was not focused on any clinical outcome, was selected as the best biomedical publication of 2002 by The Lancet.
An enlarged HGDP collection would enhance research on the history and structure of human populations from all parts of the world, allowing researchers to characterize the global variation in any genetic sequence of interest. This work is useful to both evolutionary and medical studies. Identifying the genetic bases of complex diseases (the focus of the HapMap project) and of variation in response to environmental exposures and to drugs requires an understanding of genomic variation among unaffected individuals (controls) of similar ancestry to affected individuals.

Defining appropriate control populations for modern genomic analysis is still in its infancy. It can be helped by continuing political and financial support for HGDP resources.

Luigi Luca Cavalli-Sforza

Department of Genetics,

Stanford University School of Medicine,

Stanford, California 94305, USA

\section{Small change}

Sir - Changes to the way in which the Medical Research Council (MRC) distributes funds in the United Kingdom are indeed welcome, as is any increase in the medical research budget (Nature 427, $665 ; 2004)$. However, to put the latter point into perspective, the entire MRC budget is not much more than the increase of US\$729 million proposed by President George Bush for the US National Institutes of Health budget in fiscal year 2005. On this point, the best one can say is that a very small pie is now merely small. Stephen E. Moss

Division of Cell Biology, Institute of Ophthalmology, University College London, Bath Street,

London EC1V 9EL, UK

\section{Irrelevance, buzzwords, coffee: it's the virtual PI}

Sir - Several graduate students would like to suggest a modest proposal to the authors of the recent article "Functional genomic hypothesis generation and experimentation by a robot scientist" (Nature 427, 247-252; 2004).

We've been doing a little research of our own and have generated an exciting hypothesis: we can build a Virtual Principal Investigator.

The software platform will consist of a tiny amount of background knowledge about a completely different system from the one being actively investigated, a buzzword-invention engine, coffeeconsumption apparatus, a grant-writing node and a cat-o'-nine-tails that will tie everything together.

For much less money than an actual investigator, we created a machine that resides in an office, drinking coffee at a steady rate. At bi-monthly intervals, the Virtual Principal Investigator spits out grants that are derivatives of highly successful grants from the past. Between these time points, it will play an endless loop of inspirational messages and test the effectiveness of the whip as a method of controlling subservient organisms.

Initial tests of our Virtual Principal Investigator have revealed no differences from our existing model of an investigative scientist, but we have yet to perform an experiment that was not naive or random. We look forward to discussing our results with you.

Several non-robot scientists (names supplied)

Department of Biological Chemistry and Molecular Pharmacology, Harvard Medical School,

240 Longwood Avenue, Boston,

Massachusetts 02115, USA 